\title{
SALIR DEL LOTO Y CAER EN EL ARROYO! $\left({ }^{\star}\right)$.
}

Il signor Alberto Kraft ha voluto dir la sua sulla polemica cui ha dato origine il "trucco" operato dal signor Levillier sopra un trozo del notissimo planisfero Hamy. Ma questa spiegazione, che giunge ad oltre quattro anni di distanza dalla mia prima segnalazione, non spiega nulla; anzi, caso mai nel caso cioé che potesse essere tenuta per veritiera -- mentre non libera il signor Levillier dalle sue responsabilità, aggrava quelle della Casa Editrice, la "Guillermo Kraft Ltda, Soc. An. de Impresiones Generales" di Buenos Aires.

Ed eccone le ragioni.

Per quanto riguarda la Casa, si deve osservare che: 1) sebbene il sig. A Kraft non precisi, come avrebbe dovuto, di qual originale la Casa si servi per la riproduzione del planisfero, si deduce dalle sue parole che fu utilizzata a questo scopo una riproduzione, a stampa, di una riproduzione autotipica. Quale meraviglia, quindi, che una riproduzione fotografica della riproduzione d'una riproduzione, risultasse tecnicamente inadeguata? Meraviglia piuttosto che non si sia giudicata tale subito, prima di eseguire la fototipia destinata al volume; 2) - il signor A. Kraft tiene a far risaltare lo serupolo col quale la Casa editrice selezionava le fotografie. Si é visto quanto fosse puntuale questo scrupolo. Ma non é tutto. Non c'é stampatore non sappia una carta geografica manoscrita non é la fotografia di un paesaggio, od un ritratto, o un disegno di fantasia, su cui si possano impunemente mettere le mani. La carta geografica é un documento, nel quale ogni segiro, e soprattutto le indicazioni relative alle coordinate di posizione, deve essere davvero scrupolosamente rispettato. Le cifre che si vollero esclarecer non andavano toccate, tanto più che, data la divisione in un certo numero di segmenti uguali indicata sui due margini sinistro e destro della carta, bastavano anche

(*). - Rssolvemos publicar em seu próprio texto, sem traduçāo, a resposta dada pelo Prof. Giuseppe Caraci à carta editada por esta Revisia (n. ${ }^{\circ} 35$, pág. 147), da autoria do Dr. Alberto Kraft, por se tratar de polêmica (Nota div Redasaáa). 
due sole cifre leggibili per ricavare senza errori tutte le altre. E. la riproduzione, per quanto scadente, si trovava certo in queste condizioni, come si deduce dalla stessa tavola truccata; 3) - l'unica soluzione ch'era da adottare, dati gli scrupoli di cui parla il signor Kraft, sarebbe stata quella di gettar via la riproduzione utilizzata, e di procurarsene una soddisfacente. Né la cosa era difficile, avendosi diverse buone riproduzioni del planifesro in questione.

Purtroppo, invece, provvedimenti di questo genere non dovettero esserne presi quanti pur sarebbero stati necessari dalla Casa editrice, non ostante gli serupoli di cui parla il signor A. Kraft. Difatti, per limitarmi al II volume dell'America la bien llamada, dove é inserita la carta truccata, l'attento lettore potrà osservare che, delle riproduzioni, si salvano, tecnicamente, quasi soltanto quello di carte a stampa. Le fototipie delle carte manoscritte sono, nella loro grande maggioranza, ricavate da originali scadenti, o da riproduzioni sfocate, scure o tecnicamente non riuscite. Cito qui, a riprova, quelle introdotte nelle pagine $10,19,21,28,29,46,53,68,77,109,112,117$, $119,144,214,215,218,222,228, \ldots$ e mi pare che basti (controlli chi vuole).

$\mathrm{E}$ veniamo ora al signor Levillier. A proposito del quale non posso non rilevare prima di tutto come il signor A. Kraft mi attribuisca, nei confronti del suo autore, un extraño ensanamento, che, a suo avviso, toglierebbe consistencia ai miei rilieve. Hi basterà invitare il signor $\mathrm{A}$. Kraft a rileggere le pagine con le quali il signor Levillier credette di poter rispondere alle critiche da me fatte sull'A. la b.11.; e dopo si persuaderà da qual parte stia l'estraño ensañamento.

Ma torniamo a bomba.

1) - stando alle dichiarazioni dal signor A. Kraft, il signor Levillier, interpellato, mentre era a Londra, sul modo di risolvere la questione postagli a proposito del planisfero Hamy, avrebbe richiesto alla Casa editrice che venissero rinforzate le cifre indicanti le latitudini sulla sinistra del disegno. Ma, riconosce lo stesso signor $\mathrm{A}$. Kraft, mentre sarebbe stato necessario che venissero date istruzioni precise, "é da lamentare" - sono sue parole - "che il signor Levillier non ci dicesse in qual modo dovessimo tradurre in atto la sua richista". Mi sembra che non ci sia bisogno di commenti: lo scrupolo del signor Levillier avrebbe avuto, ancor piu che le cifre del trozo, bisogno di un adeguato rinforzo! 2) - la soluzione sarebbe dunque stata rimessa al disegnatore; il quale avrebbe operato come si é visto. Ma se cosi fosse avvenuto veramente, come mai 
ai suo ritorno in Argentina, il signor Levillier non si sarebbe accertato se la soluzione stessa andasse, o non, d'accordo col testo destinato ad illustrare il planisfero? Tra la fine del 1947 - quando la Casa editrice si accorse che la riproduzione in oggetto era inadeguata - ed il 30 ottobre 1948 - quando il secondo volume dell'A la B. 11 . vide la luce (cfr. l'avvertenza in fine del volume stesso) - passarono almeno 10 mesi. $\mathrm{Ci}$ scirebbe stato dunque, tutto il tempo di provvedere; ma di fatto non risulta si sia tentato nulla.

Anche questa, allora, una prova dello serupolo congiunto della Casa editrice e dell'autore del volume? 3) - ma ammettiamo pure che in quei dieci mesi di tempo la tipografia non potesse porre rimedio all'inconveniente (sebbene se ne dovrebbe concludere in modo ron certo molto lusinghiero per la sua attrezzatura tecnica!); ciò non avrebbe toito al signor Levillier la possibilità di mettere in chiaro l'operato del disegnatore, magari mediante una breve nota.

Non avendolo fatto, egli autorizzerebbe plienamente - anche se potessimo dar credito alle dichiarazioni del signor A. Kraft - le illazioni contenute nel mio scritto: Areo que mucho brega, ecc., inserito in questa "Revista". E'le autorizzerebbe, appunto perché la riproduzione del - trovo - non poteva in alcun modo essere fine a sé stessa, quasi si trattasse di un particolare meramente esornativo, come si fa nelle riviste a rotocalco, ma doveva servire a legittimare le conclusioni cui lo stesso Levillier ha creduto di poter giungere sopra uno dei problemi fondamentali per la sua ricerca, vale a dire quello del punto più mexidionale toccato dal Vespucci nella spedizione del 1501-1502.

$\mathrm{Ne}$ segue che la posizione di distratto agnosticismo assunta dal signor Levillier, di fronte alla richiesta di chiarimenti rivoltagli a Londra dalla Casa editrice, col rimettere sic et simpliciter la soluzione al disegnatore, non farebbe che confermare appieno le riserve da me fatte così sulla tesi dei due volumoni, come sulla capacità specifica del loro autore ad occuparsi di cartografia nautica. E infatti:

a) la differenza di $5^{\circ}$ in più o in meno di latitudine porta a conseguenze ben diverse per la tesi del signor Levillier, e rende semplicemente inconcepibile che questi non se ne fosse reso conto prima - e non dopo - avere dato corpo alla tesi stessa, tenuto anche conto dell'importanza tutta speciale che, per la ricostruzione di quel viaggio, presenta per l'appunto il planisfero in questione; 
b) anche prescindendo dall'assurdo sopra esposto, non si potrebbe non rimanere sorpresi che una volta vedute le alterazioni apportate dal disegnatore, il "competente", che avrebbe dovuto essere il signor Levillier, non si fosse né meno accorto che le cifre alterate segnano, a $\mathrm{N}$., due volte il $5^{\circ} \mathrm{N}$. ed a S. due volte il $40^{\circ} \mathrm{S}$, e non indicano l'Equatore!! Insomma, volendo tutto salvare, l'intervento del signor $\mathrm{A}$. Kraft non fa, come dicevo, che rendere sempre più grave starei per dire più comica - la posizione del signor Levillier; 4) - un dato di fatto sicuro, che non ha bisogno delle dichiarazioni del signor A. Kraft, é questo: il signor Levillier era fermamente convinto che l'alterazione delle cifre, anche se dovuta a iniziativa del cartografo, rispondeva ad una esigenza a suo credere giusta, tanto é vero che poté scrivere che il disegnatore aveva emendato un errore, mentre era proprio lui a commettere un errore, e dei più marchiani! Particolare significativo: infatti soltanto con questa soluzione si dava un'ombra di legittimità alla tesi da lui sostenuta .

Concludendo: la verità é nelle mani di Dio, ma sia stato, o non, intenzionale il "trucco", sia esso attribuibile alla Casa editrice, al disegnatore od il signor Levillier, non v'ha dubbio che la responsabilità finale va attribuita alla persona sotto il cui nome l'opera é pubblicata. E' infatti l'autore, e lui soltanto, che col "si stampi", dà fine al lavoro dell'editore, e riserba a sé meriti e demeriti di ciò che si stampa. Ne segue che l'interpretazione da me data resta una delle due sole possibili che, dagli elementi in mio possesso, mi sembrò - e mi sembra ancora - la più logica e la più persuasiva.

Quanto all'altra, quella offerta ora, a quattro anni di distanza dai miei rilievi ed a dodici dagli eventi a cura dle signor A. Kraft, giova rimettersi all'opinione del lettore. Di fatto, chi scrive non avrebbe altro motivo valido, oltre le ragioni che si son dette, per oppognarla, al di fuori di quello sopra esposto: accettando una simile interpretazione, il signor Levillier ne risulta squalificato a vita come cultore di questioni cartografiche. E' infatti evidente che non poteva giudicarne a ragion veduta che, ancora sei anni dopo la composizione dei due volumoni, mostrava di ignorare non si dice l'uso del doppio equatore, ma fin la normale sequenza delle cifre di latitudine sul margine di una carta geografica: ignoranza, questa, che non si perdonerebbe neppure ad uno scolaretto delle scuole elementari! 